\title{
Collapse suppression and soliton stabilization through nonlocality in bulk Kerr media
}

\author{
Bang, Ole; Chemineau, E. T.; Krolikowski, Wieslaw
}

Published in:

Nonlinear Optics: Materials, Fundamentals and Applications

Link to article, DOI:

10.1109/NLO.2000.883662

Publication date:

2000

Document Version

Publisher's PDF, also known as Version of record

Link back to DTU Orbit

Citation (APA):

Bang, O., Chemineau, E. T., \& Krolikowski, W. (2000). Collapse suppression and soliton stabilization through nonlocality in bulk Kerr media. In Nonlinear Optics: Materials, Fundamentals and Applications (pp. 322-324). https://doi.org/10.1109/NLO.2000.883662

\section{General rights}

Copyright and moral rights for the publications made accessible in the public portal are retained by the authors and/or other copyright owners and it is a condition of accessing publications that users recognise and abide by the legal requirements associated with these rights.

- Users may download and print one copy of any publication from the public portal for the purpose of private study or research.

- You may not further distribute the material or use it for any profit-making activity or commercial gain

- You may freely distribute the URL identifying the publication in the public portal 


\title{
Collapse suppression and soliton stabilization through nonlocality in bulk Kerr media
}

\author{
Ole Bang and Eric T. Chemineau \\ Department of Mathematical Modelling, Technical University of Denmark, \\ building 305, 2800 Kongens Lyngby, Denmark \\ Tel.: (+45) 452531 08; Fax: (+45) 45931235 ; E-mail: ob@imm.dtu.dk \\ Wieslaw Krolikowski \\ Australian Photonics Cooperative Research Centre, Laser Physics Centre, \\ Research School of Physical Sciences and Engineering, \\ Australian National University, Canberra ACT 0200, Australia. \\ Tel.: (+61) 26249 3752; Fax: (+61) 26249 0029; E-mail: \\ wzk111@rsphysse.anu.edu.au
}

\begin{abstract}
We show that self-focusing cannot occur in bulk Kerr media with a nonlocal nonlinear response. We find the stationary solutions and show that nonlocality makes them stable. The results are verified numerically.

(C) 2000 Optical Society of America

OCIS codes: (190.3270) Kerr effect; Pulse propagation and solitons
\end{abstract}

Consider the propagation of a localized beam with slowly-varying envelope function $u=u(\vec{r})=u(\vec{r}, z)$ in a nonlocal Kerr medium. When diffracting in two transverse dimensions (2D) the evolution of such beams can be described by the integro-differential equation $[1,2]$

$$
i \partial_{z} u+\nabla^{2} u+N(I) u=0, \quad N(I)=\int R\left(\vec{r}^{\prime}-\vec{r}\right) I\left(\vec{r}^{\prime}\right) d \vec{r}^{\prime},
$$

where $I=I(\vec{r})=I(\vec{r}, z)=|u|^{2}$ is the intensity, $z$ is the propagation coordinate, $\vec{r}=(x, y)$ is the transverse coordinate, and $\int d \vec{r}$ denotes $\iint_{-\infty}^{\infty} d x d y$. The nonlocal response $N=N(I)$ has the general phenomelogical form of a convolution integral, where the response function $R(\vec{\Delta})$ is assumed to be real, symmetric, localized, and positive definite. This model describes real physical situations where the nonlinear response involves transport effects such as heat conduction in materials with thermal nonlinearity [3], diffusion of molecules or atoms accompanying light propagation in atomic vapours [4] and plasma [5]. Also this form of nonlocal response appears as a result of many body interaction processes in the description of Bose-Einstein condensates [6].

In the limit when the response function becomes a delta-function, $R(\vec{\Delta})=\delta(|\vec{\Delta}|)$, the nonlinear response is local and simply given by $N(I)=I$ as in local Kerr media described by the standard nonlinear Schrödinger (NLS) equation. In this local limit it is well-known that multidimensional beams with a power higher than a certain critical value, will experience unbounded self-focusing and collapse after a finite propagation distance. In the opposite limit of a highly nonlocal response much wider than the beam width Snyder and Mitchell showed that Eq. (1) becomes the simple equation for a linear harmonic oscillator [7], which evidently does not possess any collapse singularities. The stabilizing effect of nonlocality in Kerr media was studied already in 1985 by Turitsyn [1]. For a few specific physical examples, such as Coulomb interaction $[V(\vec{\Delta})=1 /|\vec{\Delta}|]$, Turitsyn showed that the Hamiltonian was bounded from below for fixed power, and thus that the stationary ground-state solitary wave solutions (solitons) are stable in a Lyapunov sense.

Here we consider the weakly nonlocal limit, in which the width of the response function is small on the scale of the incident beam width. We show that any nonlocal response then reduces to the Kerr nonlinearity plus a diffusion-like term, $N \sim I+\nabla^{2} I$, as also discussed by Suter and Blasberg [4]. We use this to prove rigorously that collapse is completely suppressed by nonlocality, irrespective of the form of the nonlocal response. We further find the soliton solutions using collective coordinates, also in the general case, and we show that the solitons are stable in the local linear Vakhitov-Kolokolov sense. Finally we confirm the stability numerically and show how the evolution of the 2D NLS soliton is affected by the degree of nonlocality.

For localized or periodic solutions Eq. (1) conserves the power $P$, the Hamiltonian $H$, and the momentum in the $x$-direction $M_{x}$ and the $y$-direction $M_{y}$,

$$
P=\int I d \vec{r}, \quad H=\int\left\{|\nabla u|^{2}-\frac{1}{2} N I\right] d \vec{r}, \quad M_{x, y}=\int 2 \operatorname{Im}\left\{u^{*} \partial_{x, y} u\right\} d \vec{r} .
$$


To analyze the dynamics we define the mean square beam width or so-called virial, $V(z)=P^{-1} \int r_{0}^{2} I d \vec{r}$, where $\vec{r}_{0}=\vec{r}-\langle\vec{r}\rangle$ is the distance from the center of mass $\langle\vec{r}\rangle=P^{-1} \int \vec{r}|u(\vec{r})|^{2} d \vec{r}$ and $r_{0}=\left|\vec{r}_{0}\right|$. Using the continuity equations for the conserved quantities we obtain for localized solutions the first derivative $d V / d z=4 P^{-1} \int \operatorname{Im}\left\{\vec{r}_{0}\right.$. $\left.u^{*} \nabla u\right\} d \vec{r}$, and the virial relation for the acceleration

$$
\frac{d^{2} V}{d z^{2}}=\frac{8}{P}\left(H-\frac{M^{2}}{4 P}-G(I)\right), \quad G(I)=\frac{1}{2} \int\left[N I+\left(\vec{r}_{0} \cdot \nabla I\right) N\right] d \vec{r} .
$$

In the local limit when $N(I)=I$ the nonconserved part vanishes, $G(I)=0$, and thus Eq. (3) reduces to the well-known virial relation for the standard NLS equation. We have not been able to reduce the general virial relation (3) to a form that includes only conserved quantities or is boundable. To proceed we therefore make the simplifying assumption that the response function is narrow compared to the intensity distribution, i.e., the nonlinear response is only weakly nonlocal, as sketched in Fig. 1(left).
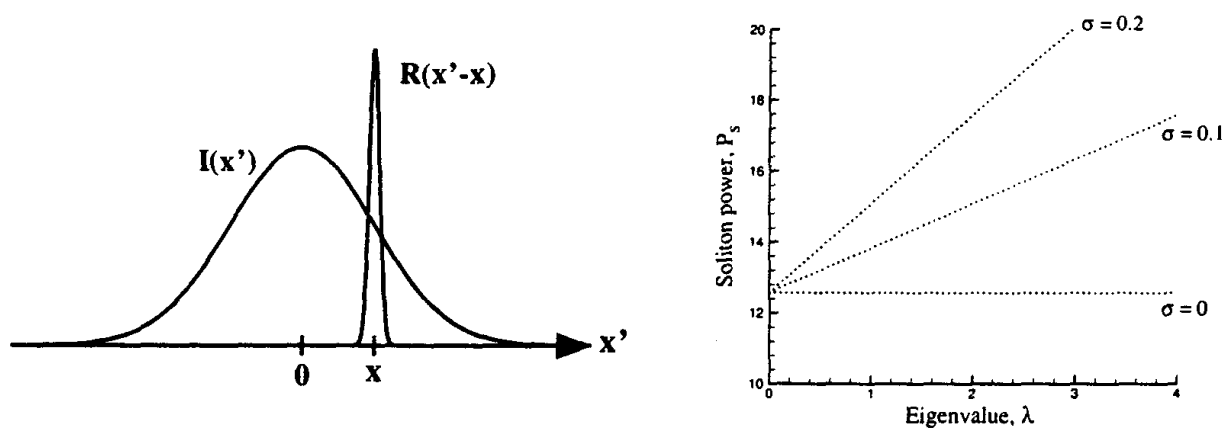

Fig. 1. Left: $x^{\prime}$-plane of the intensity profile $I\left(\vec{r}^{\prime}\right)$ and response function $R\left(\vec{r}^{\prime}\right)$ in the weakly nonlocal limit. Right: Soliton power $P_{s}=4 \pi\left(1+\sigma^{2} \lambda\right)$ versus the eigenvalue $\lambda$ for different degrees of nonlocality $\sigma$ calculated from the weakly nonlocal Gaussian variational solutions.

When $R(\vec{\Delta})$ is so localized that $I\left(\vec{r}^{\prime}\right)$ varies only slightly where $R(\vec{\Delta})$ is different from zero, then we can expand $I\left(\vec{r}^{\prime}\right)$ in a Taylor series around $\vec{r}^{\prime}=\vec{r}$ and obtain the reduced response function

$$
N(I)=\gamma_{0} I+\gamma_{2} \nabla^{2} I, \quad \gamma_{0}=\int R(\vec{r}) d \vec{r}, \quad \gamma_{2}=\frac{1}{4} \int r^{2} R(\vec{r}) d \vec{r},
$$

where the parameters $\gamma_{0}$ and $\gamma_{2}$ are positive definite. When $R(\vec{r})$ is a delta function $\gamma_{0}=1$ and $\gamma_{2}=0$, and we recover the local Kerr nonlinearity. Thus $\gamma_{2}$ is a small parameter for the weakly nonlocal case. For this weakly nonlocal response the nonconserved integral $G(I)$ is sign definite and can be calculated explicitly resulting in the simplified virial relation

$$
\frac{d^{2} V}{d z^{2}}=\frac{8}{P}\left(H-\frac{M^{2}}{4 P}+\frac{\gamma_{2}}{2} \int|\nabla I|^{2} d \vec{r}\right) \geq \frac{8}{P}\left(H-\frac{M^{2}}{4 P}\right) .
$$

From the bound we see that $H-M^{2} /(4 P)>0$ is a sufficient condition for the absence of collapse. However, we can also look directly at the virial relation (5): For a given initial condition the conserved quantities are fixed. Let us then assume that the right hand side is initially negative, so that the beam width will start to decrease. Can a collapse then develop? No, since then the last integral would diverge to plus infinity, meaning that $V_{z z}$ would become plus infinity and therefore the beam would instantaneously diffract completely. This shows that even a small nonlocality will completely stop collapse from occuring in the mathematical sense, as also found by Davydova and Fishchuk [5].

Consider the stationary ground-state solutions of the system, which have the form

$$
u(\vec{r}, z)=\phi(r) \exp (i \lambda z),
$$

where the profile $\phi(r)>0$ is real, positive, and radially symmetric and the eigenvalue $\lambda>0$ is positive. For weakly nonlocal responses given by Eq. (4) the dynamical equations for the ground-state solutions can 
be derived from the Lagrangian $L_{s}=\int_{0}^{\infty} r\left[\lambda \phi^{2}+\phi_{r}^{2}-\gamma_{0} \phi^{4} / 2-\gamma_{2} \phi^{2}\left(\phi_{r}^{2}+\phi \phi_{r r}+r^{-1} \phi \phi_{r}\right)\right] d r$, where subscript denotes differentiation. Applying the variational approach we look for stationary solutions of the form $\phi(r)=a_{0} f\left(r / b_{0}\right)$, where $a_{0}$ and $b_{0}$ are $z$-independent collective coordinates. Inserting this ansatz into the Lagrangian $L_{s}$ we obtain the reduced Lagrangian $L_{r}=\lambda a_{0}^{2} b_{0}^{2} s_{1}+a_{0}^{2} s_{2}-\gamma_{0} a_{0}^{4} b_{0}^{2} s_{3} / 2+2 \gamma_{2} a_{0}^{4} s_{4}$, where the constants $s_{n}>0$ are positive definite and given as integrals over the trial function: $s_{1}=\int_{0}^{\infty} \tau f^{2} d \tau, s_{2}=\int_{0}^{\infty} \tau f_{\tau}^{2} d \tau$, $s_{3}=\int_{0}^{\infty} \tau f^{4} d \tau$, and $s_{4}=\int_{0}^{\infty} \tau f^{2} f_{\tau}^{2} d \tau$. The Euler-Lagrange equations for $a_{0}$ and $b_{0}$ then gives

$$
a_{0}^{2}=\frac{2 \lambda s_{1}}{\gamma_{0} s_{3}}, \quad b_{0}^{2}=\frac{s_{2}}{\lambda s_{1}}+\left(\frac{\gamma_{2}}{\gamma_{0}}\right) \frac{8 s_{4}}{s_{3}}, P_{s}=P_{s}^{\mathrm{nls}}\left[\left(\frac{s_{1} s_{2}}{s_{3}}\right)+\epsilon \lambda\left(\frac{8 s_{1}^{2} s_{4}}{s_{3}^{2}}\right)\right],
$$

where the small parameter $\epsilon=\gamma_{2} / \gamma_{0} \ll 1$ measures the relative strength of the weak nonlocal contribution to the nonlinear response. $P_{s}$ is the soliton power and $P_{s}^{\text {nls }}=4 \pi / \gamma_{0}$ is the $\lambda$-independent power of the Gaussian approximation to the soliton solutions of the standard 2D NLS equation (also the collapse threshold power), which is recovered in the local limit $\epsilon=0$. Since $s_{4}>0$ for any trial function $d P_{s} / d \lambda$ is positive definite. According to the Vakhitov-Kolokolov criterion the soliton solutions are therefore linearly stable, $d P_{s} / d \lambda>0$, for any form of the nonlocal response, as long as it is weakly nonlocal. In contrast they are unstable in the standard local NLS equation, in which $d P_{s}^{\text {nls }} / d \lambda=0$.

Let us consider a general response function in the form of a Gaussian,

$$
R\left(\vec{r}^{\prime}-\vec{r}\right)=\frac{1}{\pi \sigma^{2}} \exp \left(-\frac{\left|\vec{r}^{\prime}-\vec{r}\right|^{2}}{\sigma^{2}}\right)
$$

which becomes a delta-function in the local limit when $\sigma \rightarrow 0$. For this form of the response function $\gamma_{0}=1$ and $\gamma_{2}=\sigma^{2} / 4$. Let us further specify the trial function to be a Gaussian also, $f(\tau)=\exp \left(-\tau^{2}\right)$, so that $u(\vec{r}, z)=a_{0} \exp \left[-\left(r / b_{0}\right)^{2}\right] \exp (i \lambda z)$. In the weakly nonlocal limit with $N(I)$ given by Eq. (4), this would give $a_{0}^{2}=4 \lambda$ and $b_{0}^{2}=\frac{2}{\lambda}+2 \sigma^{2}$. In Fig. 1(right) we have shown the corresponding soliton power $P_{s}(\lambda)=4 \pi\left(1+\sigma^{2} \lambda\right)$ in this weakly nonlocal limit for different degrees of nonlocality $\sigma$. However, it is straightforward to find the solution in the general case for this choice of response and trial function. Inserting Eqs. (8) and and the trial function into the general nonlinear response (1) we obtain

$$
N=\frac{a_{0}^{2} b_{0}^{2}}{b_{0}^{2}+2 \sigma^{2}} \exp \left(-\frac{2 r^{2}}{b_{0}^{2}+2 \sigma^{2}}\right)
$$

The Euler-Lagrange equations on the Lagrangrian $L_{s}=\int_{0}^{\infty} r\left(\lambda \phi^{2}+\phi_{r}^{2}-N \phi^{2}\right) d r$ for the full system with $N$ given by (9) then gives the power $P_{s}=\pi a_{0}^{2} b_{0}^{2} / 2$ with the width $b_{0}$ and amplitude $a_{0}$ given by

$$
b_{0}^{2}=\sigma^{2}\left[\frac{1}{\sqrt{1-4 \lambda / a_{0}^{2}}}-1\right], \quad \frac{\sigma^{2}}{\sqrt{1-4 \lambda / x}}-\sigma^{2}=\frac{2+\sigma^{2} \lambda}{x-2 \lambda}\left[1+\sqrt{1+4 \sigma^{2} \frac{x-2 \lambda}{\left(2+\sigma^{2} \lambda\right)^{2}}}\right]
$$

where $x=a_{0}^{2}>4 \lambda$. For $\sigma^{2} \ll 1$ the full solution approaches the weakly nonlocal solution, as it should.

We will further present the results of direct numerical simulations to verify the absence of collapse and the stability of the soliton solutions. The investigation of dark solitons in nonlocal Kerr media will be presented in a separate contribution.

We acknowledge useful discussions with J.J. Rasmussen, Y. Kivshar, and S. Turitsyn. O. Bang acknowledges support from the Danish Technical Research Council (Talent Grant no. 9800400).

\section{References}

1. S.K. Tusitsyn, Teor. Mat. Fiz. 64, 226 (1985).

2. S. Abe and A. Ogura, Phys. Rev. E 57,6066 (1998).

3. J.P. Gordon, R.C. Leite, R.S. Moore, S.P. Porto, and J.R. Whinnery, J. Appl. Phys. 36, 3 (1965).

4. D. Suter and T. Blasberg, Phys. Rev. A 48, 4583 (1993)

5. T.A. Davydova and A.I. Fishchuk, Ukr. J. Phys. 40, 487 (1995)

6. F. Dalfovo, S. Giorgini, L.P. Pitaevskii, and S. Stringari, Rev. Mod. Phys. 71, 463 (1999).

7. A.W. Snyder and D.J. Mitchell, Science 276, 1538 (1997). 